\title{
Esophageal Cancer and Bisphosphonates
}

\author{
Alexander M. Walker* \\ World Health Information Science Consultants
}

${ }^{*}$ Corresponding author: Alexander Walker, World Health Information Science Consultants, 275 Grove Street, Suite 2-400, Newton MA 02466, USA, Tel: (1) 617663 5945, E-mail: Alec.Walker@WHISCON.com

Citation: Alexander Walker (2014) Esophageal Cancer and Bisphosphonates. J Pharm Drug Devel 2(1): 101. doi: 10.15744/2348-9782.1.301

Received Date: February 3, 2014 Accepted Date: March 7, 2014 Published Date: March 11, 2014

\begin{abstract}
Background: In 2009 the FDA noted reports of esophageal cancer in persons who had received alendronate or other bisphosphonates. It called for the evaluation of these agents as risk factors.

Objective: To estimate the relative incidence of esophageal cancer in women who used oral bisphosphonates or raloxifene versus contemporaneous women of the same age.

Methods: In the General Practice Research Database, cases of esophageal cancer were compared to a subcohort of 25,000 women matched on birth year and onset date. A second, intent-to-treat analysis reformulated the GPRD records into cohorts of first-time users.

Results: 684,815 women experienced 929 cases of esophageal cancer. Users of bisphosphonates were less healthy than comparison women. Use of acid suppressants, heavy alcohol consumption and smoking raised risk. Use of bisphosphonates more than two years before clinical onset carried an adjusted RR of 1.3 (95\% CI 1.0-1.7). Alendronate carried RRs of 1.1 and 1.0. After four years from initiation of therapy, the adjusted RR fell to the null.

Conclusion: Alendronate did not increase the risk of esophageal cancer. For bisphosphonates collectively, the low level of transient increase and correlations between use and other risk factors make a causal interpretation tenuous.
\end{abstract}

\section{Introduction}

At the beginning of 2009, Dianne Wysowski of the US Food and Drug Administration summarized 23 reports of esophageal cancer following alendronate use [1]. Wysowski also noted that there had been 31 cases following alendronate use in Europe and Japan and ten cases in which other bisphosphonates had been reported as concomitant or suspect treatment. Median time from exposure to diagnosis had been 2.1 years in the US and 1.3 years in Europe. In the two series, 12 cases with histological information had adenocarcinoma and six had squamous cell carcinoma. Wysowski suggested that oral bisphosphonates should be investigated as a risk factor for esophageal cancer.

In response to Wysowski's letter, correspondents noted the short time interval between treatment and cancer diagnosis, the lack of an expected count of cases in bisphosphonate users against which to judge the number reported, the many bisphosphonate users worldwide and the voluntary nature of the FDA reporting system [2]. Two writers found lower rates of esophageal cancer in bisphosphonate users than in comparison groups in previously collected data [3,4]. As a consequence of the FDA report, we initiated research using the General Practice Research Database in the UK (now the Clinical Practice Research Datalink, or CPRD).

\section{Methods}

Study subjects were women age 55 and older with records in the GPRD from 1996 forward. The study drugs were alendronate (trade name Fosamax), etidronate (Didronel), risedronate (Actonel), ibandronate (Boniva), raloxifene (Evista) and calcitonin (Calcinar, Miacalcic, Calcitare, Forcaltonin). There being only 29 users of calcitonin among over 300,000 match set members in the case-cohort design (see the next paragraph) and no cases of esophageal cancer among them, calcitonin has been dropped from the remainder of this report. There were two analytic designs, case-cohort and intent-to-treat. In the first, cases of esophageal cancer were compared to a sample of the general population; in the second, comparison cohorts were defined by having received (or not) a first prescription for a study drug and the cohorts were followed forward for the occurrence of cancer [5]. The case-cohort study included more cases, but the intent-to-treat analysis had nearly complete chart review and was better suited for elucidating time effects and accounting for therapeutic changes. 


\section{The General Practice Research Database}

The GPRD was a repository of electronic medical records from over 600 general practices in the United Kingdom [6]. Maintained since 1987 and most recently encompassing 68 million person-years ${ }^{*}$ of history on 11 million people, the data described encounters between patients and their general practices, including prescriptions written. Under UK law, patients had the right to have data about them withheld or removed from the research database. In the UK National Health Service, information on non-GP care flows back to the general practice. The Medicines and Healthcare Products Regulatory Agency promoted and regulated research uses of the GPRD and the current CPRD [7]. This study received ethical approval from the GPRD's Independent Scientific Advisory Committee.

\section{Case-cohort design}

Women born between 1922 and 1953 became eligible for inclusion on the later of January 1, 1996, their 55 $5^{\text {th }}$ birthdays or upon completing 720 days (that is, 24 30-day "months") in an up-to-standard practice as defined by the GPRD, through the end of 2008. Women remained eligible until death, change of GP or December 31, 2009, whichever came earliest. Otherwise eligible women were removed from the study population at first recorded receipt of chemo- or radiotherapy or upon the recording of a diagnosis of Paget's disease or esophageal cancer.

\section{Cases}

A GPRD Medical Code for esophageal cancer qualified a woman as a candidate case. For all candidates in the intent-to-treat analysis (see below), for those with insufficient electronic information for case adjudication and for a random sample of the rest, GPRD staff contacted the GP. Practices were asked to review paper and electronic data and return supporting documentation for the diagnosis of esophageal cancer, the cell type, and the probable dates of symptomatic onset. Cases for which GP review was not done were adjudicated on the basis of the EMR .

\section{Population comparison sample}

The comparison subcohort was a random sample of 25,000 drawn from the pool of all eligible women. For each case, we chose matched comparators, these being all women in the sample who met eligibility criteria on the case's onset date and who had the same year of birth.

Exposure to each study drug and the number of calendar months in which each was prescribed were noted up to 720 days before onset. First use of study drugs closer to onset ("recent initiation") was also noted, but was considered a priori to potentially reflect only pre-existing cancers and concomitant conditions.

\section{Covariates}

For each subject, we identified the characteristics listed in Table 1, using the case onset date as the reference point for all the timedependent variables. Endoscopy, alcohol consumption, smoking and BMI were lagged by the amounts indicated to avoid influence by early manifestations of as-yet undiagnosed esophageal cancers.

\begin{tabular}{|l|l|l|}
\hline Covariate & Case-cohort & Intent-to-treat \\
\hline Systemic steroids. Count of prescriptions through ... & Onset & Cohort entry \\
\hline $\begin{array}{l}\text { Reflux disease (yes/no). At least } 12 \text { dispensings of proton pump inhibitors or H2-receptor antagonists and least } \\
\text { two GPRD entries on different days bearing diagnoses of reflux disease, through ... }\end{array}$ & Onset & Cohort entry \\
\hline Reflux disease, time since. From first date at which reflux disease criteria were met, through ... & Onset & Cohort entry \\
\hline Reflux prescriptions. Number of prescriptions of proton pump inhibitors or H2-receptor antagonists through ... & Onset & Cohort entry \\
\hline Years in GPRD records. From first entry through ... & Onset & Cohort entry \\
\hline Endoscopy before ... & 720 days before onset & Cohort entry \\
\hline BMI. Last body mass index recorded before ... & 180 days before onset & Cohort entry \\
\hline Smoking. Counted as Current, Former or Never according to the most recent clinical note earlier than... & 180 days before onset & Cohort entry \\
\hline $\begin{array}{l}\text { Heavy Alcohol Consumption. Two or more diagnoses of alcohol dependence, its treatment or its complications } \\
\text { earlier than ... }\end{array}$ & 180 days before onset & Cohort entry \\
\hline
\end{tabular}

Table 1: Reference points for the definition of time-dependent covariates in case-cohort and intent-to-treat designs

One person observed for one year constitutes a person-year of observation. Fractional person-years are permissible. 


\section{Intent-to-treat design}

Women using a study drug entered an intent-to-treat cohort at first recorded prescription between 1996 and 2008 , provided that they possessed the eligibility criteria given above. Participation was further limited to women cared for in practices that continued to exist in 2011, to facilitate review of charts. Women who began different study drugs at different times were permitted to enter the cohort repeatedly as if they were different women. For each initiator we sought four contemporaneously study-eligible nonusers who had the same year of birth and who had exactly the same prior use of other study drugs.

\section{Analysis}

The effects of covariates and drugs on the occurrence of esophageal cancer were evaluated through matched logistic regression in the case-cohort analysis. In the intent-to-treat analysis, a proportional hazards regression model was used, with stratification on year of birth, calendar quarter of cohort entry, and exposure history. Both analyses yield an estimated hazard ratio, or instantaneous relative risk, as a measure of association. In both, we used a robust variance estimator to account for the participation of individuals in multiple matched sets or cohort strata [8]. The design thus follows Hernán and colleagues' proposal to account for exposure changes in an intent-to-treat analysis by allowing a subject new cohort entry without removal from a previously entered cohort [9-11].

To derive a baseline model for each analysis, we examined all of the covariates simultaneously, plus recent initiator status for each of the study drugs, and used backwards selection with a retention criterion of $\mathrm{p}<0.2$. Estimated effects for "All Bisphosphonates" were based on coefficients from models that included covariates and individual study drugs. The summary hazard ratio estimate was based on the inverse-variance-weighted mean of the drug-specific coefficients on the scale of natural logarithms. (Covariances between these estimates were extremely small).

\section{Results}

There were 684,815 women age 55+, born 1922-1953, with more than 720 days experience and at least one of their eligible days occurring between the beginning of 1996 and the end of 2008 . 929 developed esophageal cancer, of which 159 (17.1\%) had documentation as squamous cell carcinoma, 145 (15.6\%) as adenocarcinoma (Table 2).

\begin{tabular}{|c|c|c|}
\hline & Case-Cohort & Intent-to-Treat \\
\hline Confirmed & 929 & 297 \\
\hline (\% based on GP's record abstraction) & $(33.5 \%)$ & $(79.5 \%)$ \\
\hline \multicolumn{3}{|l|}{ Cell type } \\
\hline Squamous cell carcinoma & 159 & 109 \\
\hline Adenocarcinoma & 145 & 87 \\
\hline Mixed/other & 15 & 12 \\
\hline Not documented & 610 & 89 \\
\hline \multicolumn{3}{|l|}{ Calendar year of onset } \\
\hline 1996-1999 & 84 & 3 \\
\hline $2000-2004$ & 316 & 75 \\
\hline $2005-2009$ & 529 & 219 \\
\hline
\end{tabular}

Table 2: Cases of esophageal cancer among women aged 55+ in the GPRD 1996-2009

\section{Case-cohort study}

The 25,000 women in the random sample provided 338,911 comparison woman-days for the risk sets of the 929 cases. The risk sets were defined by observation date, and were matched on birth year.

Potential confounders were those characteristics that were more prevalent in esophageal cancer patients than in non-patients and were also more prevalent among users of bisphosphonates than nonusers. Table 3 describes the patients and the comparators at the time of cancer onset. Cases and the women in comparator sample had different prevalences of most covariates, with recent initiation of study drugs, endoscopy, reflux disease and its treatment, smoking, prior use of systemic steroids, indicators of alcohol-associated disease and low BMI being more common in cases. 
See Appendix Table A1 for the relations between drug use and covariates. By comparison to nonusers in the comparison sample, those who received bisphosphonates (and to a smaller degree, raloxifene) had higher prevalence of prior endoscopy, reflux disease and its treatment, former but not current smoking, use of systemic steroids and low BMI.

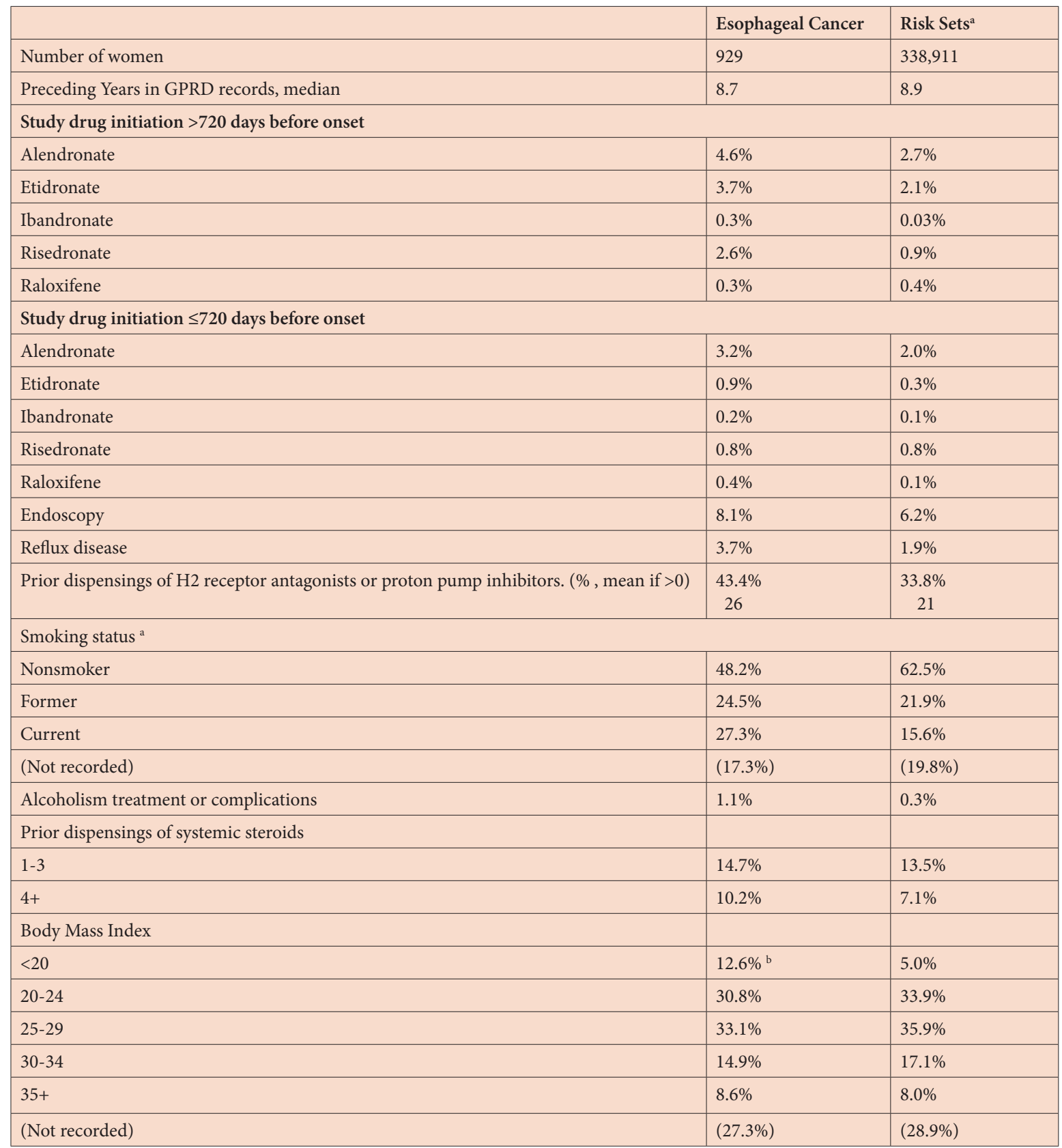

${ }^{a}$ Comparison woman-days, matched by date and year of subject's birth to cases

${ }^{b}$ Percentages are among those with recorded values

Table 3: Characteristics of esophageal cancer cases and the comparison sample

With adjustment for the possible confounding factors, exposure at least two years previously, estimated for all bisphosphonates together, was associated with a hazard ratio of 1.3 (Table 4; 95\% CI 1.0-1.7) for the occurrence of esophageal cancer. There were elevated hazard ratios associated with increasing use of anti-reflux medications, heavy alcohol use, very low BMI and smoking. Recent initiation of both etidronate and raloxifene was associated with increased risk. Appendix Table A2 presents results by cell type. Cases recorded as adenocarcinoma and those recorded as squamous cell carcinoma had risk elevations that were similar to one another with respect to the study drugs.

Per protocol, the initial modeling of exposure had included both indicator terms for study drugs and the number of calendar months in which each study drug had been prescribed. In no case did inclusion of the months of use add significantly to the prediction of cancer. To further evaluate this, we examined the distribution of study drug exposures for different times prior to onset, and found no trends that might suggest a need to account for the timing or the amount of bisphosphonate use. 


\begin{tabular}{|c|c|c|}
\hline & \multicolumn{2}{|c|}{ Hazard Ratios and $95 \%$ Confidence Intervals } \\
\hline \multicolumn{3}{|l|}{ Study Drug $>720$ days before onset } \\
\hline Alendronate & 1.1 & $0.7-1.5$ \\
\hline Etidronate & 1.3 & $0.9-2.0$ \\
\hline Ibandronate & 4.9 & $1.4-16.7$ \\
\hline Risedronate & 1.6 & $1.0-2.5$ \\
\hline All Bisphosphonates & 1.3 & $1.0-1.7$ \\
\hline Raloxifene & 0.7 & $0.2-2.2$ \\
\hline \multicolumn{3}{|l|}{ Other covariates } \\
\hline \multicolumn{3}{|c|}{ First use of Study Drug $\leq 720$ days before onset } \\
\hline Etidronate & 2.3 & $1.1-4.6$ \\
\hline Raloxifene & 3.1 & $1.1-8.7$ \\
\hline Doubling in Reflux Rx's & 1.08 & $1.05-1.12$ \\
\hline Alcoholism and its consequences & 3.2 & $1.6-6.5$ \\
\hline \multicolumn{3}{|l|}{ Body Mass Index } \\
\hline$<20$ & 2.3 & $1.7-3.0$ \\
\hline $20-24$ & 1.0 & reference \\
\hline $25-29$ & 1.0 & $0.8-1.2$ \\
\hline 30-34 & 1.0 & $0.8-1.3$ \\
\hline $35+$ & 1.3 & 0.91 .7 \\
\hline Not recorded & 1.1 & 0.91 .3 \\
\hline \multicolumn{3}{|l|}{ Smoking } \\
\hline Never & 1.0 & reference \\
\hline Former & 1.4 & $1.1-1.6$ \\
\hline Current & 2.3 & $2.0-2.8$ \\
\hline Not recorded & 1.2 & 0.91 .5 \\
\hline
\end{tabular}

Table 4: Study drug exposures and other predictors

\section{Intent-to-treat study}

There were 78,360 cohort entrants using study drugs and 300,610 comparators, with 297 cases producing 391 cohort events, for an overall incidence rate of 32 per 100,000 per year (The larger number of events results from subjects who were in more than one cohort at the time of cancer onset. The person-time is correspondingly expanded.) Differences in the composition of the treated and comparison cohorts at the beginning of treatment (Appendix Table A3) were similar to the relations between treatments and covariates seen cross-sectionally at the time of disease onset (Appendix Table A1). The relative risks associated with study drugs in the intent-to-treat analysis (Appendix Tables A4a-A4d) showed no pattern of increasing relative risk with increasing duration of follow-up. Follow-up beginning at 721 days from cohort entry (Appendix Table A4c) provided the exposure measure that corresponded to that used in the case-cohort study. The results were essentially identical to those of the case-cohort study. Follow-up beginning at 1442 days (Appendix Table A4d) showed no elevation of risk.

\section{Assessment}

The original case series reported by Wysowski raised the possibility that alendronate and other bisphosphonates might increase the incidence of esophageal cancer within a short time from first use. In the present study, two approaches to the analysis of esophageal cancer find no increase in risk associated with alendronate. For all bisphosphonates together, the data point to a risk elevation after two years on the order of 30 percent. Raloxifene use was associated with an immediate increase in diagnosis, but no longer-term rise. Raloxifene, which is not a bisphosphonate, does not irritate the esophagus and may have been a preferred treatment in persons with symptoms from what would later be recognized as esophageal cancer.The two study designs employed provide complementary lines of evidence. The case-cohort design offers more statistical power through the inclusion of more cases. Knowledge of starting time in the intent-to-treat analysis permits the separation of risks as a function of time, and it offers a way to avoid inappropriate statistical control for so-called intermediate variables, patient characteristics that might be a result and not a cause of treatment. With a smaller number of study subjects in the intent-to-treat analysis, it was possible to seek GP review of every case. 
Earlier studies from the GPRD, covering largely the same years and cases as the present work, have had inconsistent results [1214]. The first two of these reports in the GPRD had conclusions that were interpreted as discrepant, though they were in fact statistically similar, one finding an RR of 1.3 overall, as here, but with risk concentrated in longest-term users, the other finding an RR of 1.1, with no dose-response [12,13]. The third, a case-control study, reported a relative risk of 1.4 [14]. None of these studies included chart review, all accepted first computer-recorded date of cancer as the date of onset, and all effectively considered exposures right up through the date of onset. We have previously shown that onset date was not well recorded for esophageal cancer in the GPRD, some diagnoses even appearing around the time of death in patients who had clearly been under care for their cancers for some time [15]. The anomalous results with raloxifene in the present study (high relative risk with recent initiation, quickly disappearing) supports the idea that exposure patterns in the period before diagnosis may be seriously disrupted by early symptomatology. Dixon and Solomon have suggested that the difference in risk estimates could stem from different look-back periods, and maintained that harm present at the levels reported might be acceptable in comparison to the benefit of bisphosphonates in the prevention of fracture [16]. Unlike other work in the GPRD, the general practices that cared for the patients adjudicated a large number of this study's potential cases. With access to hard copy files, the GPs could date cancer onset with greater precision than the electronic record permits, and they often provided cell type [15].

Just as earlier analyses in the GPRD gave divergent results, so have studies that used Danish national records. Abrahamsen and colleagues used prescription, hospital and mortality data to examine 30,606 women age 50 and older that received alendronate from 1996 through 2005 [17]. By comparison to a fourfold larger comparison group, alendronate users experienced no higher risk of esophageal cancer through a median follow-up of 3.5 years. In contrast, using apparently the same data sources, Vestergaard identified 55,090 women who used alendronate for a mean of 2.8 years and 39,724 who used etidronate (5.5 years) [18]. Women in both groups experienced a nearly two-fold increase in risk of esophageal cancer over controls.

Points of special concern in conflicting results from the same data relate to study design. The most tractable questions relate to onset timing. Since bisphosphonates can irritate the lower esophagus, they may lead to early evaluation and diagnosis of pre-existing esophageal tumors. Physicians may also selectively avoid giving these drugs to people who already have esophageal symptoms, including people at high risk for esophageal cancer because of reflux disease and possibly some with undiagnosed esophageal cancer. The resulting tangle makes risk in the first years of bisphosphonate use uninterpretable in observational studies, and it was for this reason that we focused from the beginning on cancers occurring at least two years after first drug use.

These analyses do not provide support for the initial concern that alendronate might increase the risk of esophageal cancer. For all bisphosphonates taken together, the low level of increase, the absence of temporal effects, and demonstrated correlations between bisphosphonate use and other risk factors make a causal interpretation tenuous.

\section{Acknowledgements}

Deborah Hennessey, John Seeger and Lisa Weatherby of WHISCON provided consultation, review and operational support. Robert Lubwama of Merck oversaw contacts with GPRD, coordinated chart abstraction requests and provided the original GPRD data files and chart abstraction results to WHISCON. IMS staff Seth Goodman, Malcolm Rucker, Dan Smith and Jason Yeaw managed the data and performed analyses under WHISCON direction.

This work was carried out under a research agreement between World Health Information Science Consultants (WHISCON) and Merck \& Co., Inc.

\section{Click here to download Appendix File}

\section{References}

1. Wysowski DK (2009) Reports of esophageal cancer with oral bisphosphonate use. N Engl J Med 360: 89-90.

2. Hofbauer LC, Miehlke S, Robins HI, Holen KD, Shaheen NJ, et al. (2009) More on reports of esophageal cancer with oral bisphosphonate use. N Engl J Med 360: 1790-1791.

3. Abrahamsen B, Eiken P, Eastell R (2009) More on reports of esophageal cancer with oral bisphosphonate use. N Engl J Med 360: 1789.

4. Solomon DH, Patrick A, Brookhart MA (2009) More on reports of esophageal cancer with oral bisphosphonate use. N Engl J Med 360: $1789-1790$.

5. Prentice RL (1986) A case-cohort design for epidemiologic cohort studies and disease prevention trials. Biometrika 73: 1-11.

6. General Practice Research Database (2012).

7. Welcome to CPRD - Clinical Practice Research Datalink 2012.

8. Barlow WE (1994) Robust variance estimation for the case-cohort design. Biometrics 50: 1064-1072. 
9. Hernán MA, Alonso A, Logan R, Grodstein F, Michels KB, et al. (2008) Observational studies analyzed like randomized experiments: an application to postmenopausal hormone therapy and coronary heart disease (with discussion). Epidemiology 19: 766-779.

10. Danaei G, García Rodríguez LA, Cantero OF, Logan R, Hernán MA (2013) Observational data for comparative effectiveness research: An emulation of randomised trials of statins and primary prevention of coronary heart disease. Stat Methods Med Res 22: 70-96.

11. Hernán MA (2011) With great data comes great responsibility; publishing comparative effectiveness research in Epidemiology. Epidemiology $22: 290-291$.

12. Cardwell CR, Abnet CC, Cantwell MM, Murray LJ (2010) Exposure to oral bisphosphonates and risk of esophageal cancer. JAMA 304: 657-663.

13. Green J, Czanner G, Reeves G, Watson J, Wise L, et al. (2010) Oral bisphosphonates and risk of cancer of oesophagus, stomach, and colorectum: case-control analysis within a UK primary care cohort. BMJ 341: 4444.

14. Wright E, Schofield PT, Seed P, Molokhia M (2012) Bisphosphonates and risk of upper gastrointestinal cancer--a case control study using the General Practice Research Database (GPRD). PLoS One 7: 47616.

15. Walker AM (2011) Identification of esophageal cancer in the General Practice Research Database. Pharmacoepidemiol Drug Saf 20: 1159-1167.

16. Dixon WG, Solomon DH (2011) Bisphosphonates and esophageal cancer - a pathway through the confusion. Nat Rev Rheumatol 7: 369-372.

17. Abrahamsen B, Pazianas M, Eiken P, Russell RG, Eastell R (2012) Esophageal and gastric cancer incidence and mortality in alendronate users. J Bone Miner Res 27: 679-686.

18. Vestergaard P (2011) Occurrence of gastrointestinal cancer in users of bisphosphonates and other antiresorptive drugs against osteoporosis. Calcif Tissue Int 89: 434-441. 